\section{IVF: Wie aussagekräftig ist das Aneuploidie- Screening per DNA-Chip?}

\section{Erstmals wurde in einer klinischen Studie untersucht, wie die Ergebnisse eines Aneuploidie-Screenings per DNA-Mikroarray mit dem Implantationserfolg korrelieren.}

$\mathrm{N}$ ach den enttäuschenden Ergebnissen der Aneuploidiediagnostik per Fluoreszenz-in-situ-Hybridisierung (FISH) testen Reproduktionsmediziner derzeit die Eignung eines DNA-Mikroarrays (Affymetrix). Bei dieser Methode wird das gesamte Genom einer Zelle amplifiziert und aufgrund von Einzelnukleotid-Polymorphismen (SNPs) auf die Chromosomenzahl untersucht. In Zelllinien mit und ohne Aneuploidie konnten die US-Autoren bereits die hohe Zuverlässigkeit des Verfahrens bestätigen. In einer jetzt veröffentlichten Studie haben sie überprüft, wie gut die Testergebnisse den Implantationserfolg von IVF-Embryonen vorhersagen können.

An der Studie beteiligten sich 146 Frauen im Alter zwischen 28 und 42 Jahren, denen 255 Embryonen eingesetzt wurden. Die Embryonen wurden unmit- telbar vor dem Transfer biopsiert, entweder im Furchungsstadium ( $\mathrm{n}=113)$ oder im Blastozystenstadium ( $\mathrm{n}=142)$. Die molekulargenetische Untersuchung wurde jedoch erst zu einem späteren Zeitpunkt durchgeführt und hatte keinen Einfluss auf das weitere Vorgehen. Bei 72 Embryonen $(28,2 \%)$ war die Implantation erfolgreich.

Für 232 Embryonen konnten die Mikroarrays ausgewertet werden. Danach waren 99 Embryonen (42,7\%) aneuploid, wobei in der Hälfte der Fälle mehr als eine Chromosomenanomalie festgestellt wurde. Allerdings stellte sich heraus, dass $4 \%$ der als aneuploid klassifizierten Embryonen als gesunde euploide Kinder auf die Welt kamen. Der negative Vorhersagewert des Screenings betrug damit $96 \%$.

Von den 133 als euploid eingestuften Embryonen wurden 55 als gesunde eup-

\title{
Macht sauer inkontinent?
}

— Ob Frauen viel oder wenig Früchte mit hohem Säuregehalt essen, hat nach einer Subanalyse der Nurses' Health Study (NHS) keinen Einfluss darauf, ob sie an Harninkontinenz leiden oder nicht. Für die Studie wurden 65.168 Frauen zwischen 37 und 79 Jahren, die zunächst kontinent waren, zu ihren Ernährungsgewohnheiten befragt und vier Jahre lang beobachtet. Selbst diejenigen mit dem häufigsten Konsum an sauren Früchte und Fruchtsäften (fünf Portionen/Tag), wurden nicht häufiger inkontinent als Frauen mit dem niedrigsten Konsum (eine Portion/Tag). An diesem Null-Ergebnis änderte sich auch nichts, wenn man das Auftreten von Drang-, Belastungs- und geringfügiger Harninkontinenz einzeln betrachtete. Ebenso wenig wurde eine bestehende Harninkontinenz verschlechtert, wie die Überwachung von 23.063 bereits erkrankten NHS-Teilnehmerinnen zeigte. Eine Verschlimmerung trat innerhalb von zwei Jahren bei den Frauen mit dem höchsten und niedrigsten Konsum mit gleicher Häufigkeit auf. Die "sauren Früchte" in der Studie waren Zitrusfrüchte, Pflaumen, Trauben, Äpfel, Beeren sowie Tomaten und Tomatenprodukte. Auch bei getrennter Auswertung für die einzelnen Sorten ergaben sich keine Hinweise auf einen Zusammenhang mit der Entwicklung oder dem Fortschreiten einer Harninkontinenz. Die Autoren nennen als möglichen Grund: Die meisten säurehaltigen Früchte würden wegen ihres Gehalts an Karbonaten, Natrium und Kalium den Urin nämlich gar nicht sauer machen, sondern den $\mathrm{pH}$ sogar anheben.

Dr. Beate Schumacher

Townsend MK et al. Acidic fruit intake in relation to incidence and progression of urinary incontinence. Int Urogynecol J 2012, online 10. August; doi: 10.1007/s00192-012-1914-9

loide Kinder geboren. Der positive Vorhersagewert betrug damit zwar nur $41,4 \%$; er war aber immer noch signifikant höher als die Entbindungsrate aller Embryonen (28,2\%). Bei einer Trophektoderm-Biopsie war der positive Vorhersagewert der Untersuchung besser als bei einer Blastomer-Biopsie (48,2\% vs. $29,2 \%$ ). Für den negativen Vorhersagewert machte es dagegen keinen Unterschied, wann die Zellentnahme stattfand.

Die Chromosomenanalyse per SNPMikroarray kann nach Einschätzung der Studienautoren Aneuploidie bei menschlichen Embryonen zuverlässig erkennen. Fehlerfrei ist das Screening aber nicht: $4 \%$ der gesunden Säuglinge wurden irrtümlich als aneuploid bewertet. In einer randomisierten Studie wird jetzt überprüft, ob die Methode die Selektion von Embryonen optimieren kann.

Dr. Beate Schumacher

Scott RT et al. Comprehensive chromosome screening is highly predictive of the reproductive potential of human embryos: a prospective blinded, nonselection study. Fertil Steril 2012;97:870-5

Kommentar: Die vorliegende Arbeit von Richard T. Scott ist nach eigener Aussage die erste Studie, welche die prognostische Wertigkeit der Präimplantationsdiagnostik (PID) in einer prospektiven Studie untersucht.

Die Ergebnisse dieser Studie zeigen, dass die PID als Screening-Methode mit dem Ziel, die Chancen für ein gesundes Kind allein anhand des Chromosomenstatus zu prognostizieren, mit nicht unerheblichen Fehlern behaftet ist. Von hundert aneuploiden Embryonen würden vier fälschlicherweise aussortiert werden, würde man sich ausschließlich auf die Ergebnisse der PID verlassen. Ebenso konnte die Studie zeigen, dass alleine der euploide Chromosomenstatus keine sichere Aussage im Hinblick auf ein gesundes Kind erlaubt. Gerade bei älteren Patientinnen schränken wohl auch andere, vermutlich zytoplasmatische Faktoren die Entwicklungsfähigkeit der Embryonen ein. Auch diese Studie kann die Zweifel an der PID als allgemeine Screening-Methode nicht zerstreuen und legt nahe, die PID nur in indizierten Fällen mit z.B. monogenetischen Erkrankungen anzuwenden.

Prof. Dr. med. Thomas Katzorke 